\title{
Improving Rectification Technology in Daura Re- finery Crude Distillation Units by HYSYS
}

HYSYS تطوير تكنولوجيا تصفية النفط في وحدات التكرير باستخدام برنامج المحاكاة

\section{$\underline{\text { Abstract }}$}

It is well known that the capacity of crude distillation units in any refinery reflects the capacity of the refinery itself, and that is because we consider these units as the first stage and all other units such as vacuum distillation, hydrotreating, reforming, catalytic cracking, and others depend on them. So we devoted our effort to keep these units work with high performance and efficiency.

The purpose of this paper is to develop crude distillation units in Daura refiner, rise their performance level and overcome weak points wherever detected.

Our work here conducted by simulation program (HYSYS) and consists of two stages: Before changing operating conditions, and after changing operating conditions. Each one will be discussed separately later.

This work shows that these old units contain a lot of mistakes because they are working since 1954. These mistakes are concentrated in miss operation due to absence of daily close monitoring to the operation conditions and product specifications related to gap and overlap conditions, and this really affects badly on rectification efficiency.

\section{Introduction}

After desalting and dehydration, crude oil is separated into fractions by distillation; the distilled fractions cannot be used directly.

Another reason for complexity is that environmental legislation demands cleaner products by developing novel processes. 
Petroleum refining rocesses

\section{and operations can be sepa-}

rated into five basic areas:

- Fractionation (distillation) is the separation of crude oil in atmospheric and vacuum distillation towers into groups of hydrocarbon compounds of differing boiling-point ranges called "fractions" or "cuts".

- Conversion Processes is changing the size and/or structure of hydrocarbon molecules. These processes include:

-Decomposition (dividing) by thermal and catalytic cracking;

-Unification (combining) through alkylation and polymerization;

-Alteration (rearranging) with isomerization and catalytic reforming.

- Treatment Processes : to prepare hydrocarbon streams for additional processing and to prepare finished products. Treatment may include removal or separation of aromatics and naphthenes, impurities and undesirable contaminants. Treatment may involved chemical or physical separation e.g. dissolving, absorption, or precipitation using a variety and combination of processes including desalting, drying, hydrodesulfurizing, solvent refining, sweetening, solvent extraction, and solvent dewaxing. -Formulating and Blending is the process of mixing and combining hydrocarbon fractions, additives, and other components to produce finished products with specific performance properties.

- Other Refining Operations include:

-Light-ends recovery;

-Sour-water stripping;

-Solid waste, process-water and wastewater treatment;

-cooling, storage and handling and product movement; -Hydrogen production; 


\section{Journal of Petroleum Researches \& Studies $\quad \begin{gathered}\mathrm{N}_{\mathrm{O}} .2 \\ 2^{\text {nd. }} \text { YEAR }\end{gathered}$}

-Acid and tail-gas treatment;

-And sulfur recovery.

-Auxiliary Operations and Facili-

ties include:

-Light steam and power generation;

-Process and fire water systems;

-Flares and relief systems;

-Furnaces and heaters;

-Pumps and valves;

-Supply of steam, air, nitrogen, and other plant gases;

-Alarms and sensors;

-Noise and pollution controls;

-Sampling, testing, and inspecting and laboratory;

-Control room;

-Maintenance;

-Administrative facilities.

\section{Experimental details}

In order to perform this work as best as possible and to get real results from the simulation program (HYSYS), we did experiTable-1 analysis has been done for crude oil inlet to distillation units for different days and for the dis- tillation products before making any change in operating conditions as shown in the tables $(1,2,3,4,5)$ listed below. 
Table-1

\begin{tabular}{|c|c|c|c|}
\hline TEST & $26 / 11$ & $27 / 11$ & $30 / 11$ \\
\hline API GRAVITY @ $15.6^{\circ} \mathrm{C}$ & 35.5 & 32.9 & 32.9 \\
\hline SP.GRAVITY @ $15.6^{\circ} \mathrm{C}$ & 0.8473 & 0.8555 & 0.8555 \\
\hline \multicolumn{4}{|l|}{$\begin{array}{l}\text { SULFURE CONTENT } \\
\% \mathrm{~W} \text { W. }\end{array}$} \\
\hline KIN. VISCOSITY Cst. & 11.99 & & 17.1 \\
\hline \multicolumn{4}{|l|}{$@ 10{ }^{\circ} \mathrm{C}$} \\
\hline$@ 21.1{ }^{\circ} \mathrm{C}$ & 7.85 & 11.6 & 113 \\
\hline @ $37.8^{\circ} \mathrm{C}$ & 5.05 & & 4.8 \\
\hline$@ 50.0^{\circ} \mathrm{C}$ & 3.9 & 5.0 & \\
\hline \multicolumn{4}{|l|}{ Pour Point ${ }^{\circ} \mathrm{C}$} \\
\hline R.V.P Kg/cm2 & 0.45 & 0.47 & 0.45 \\
\hline Water \& Sediment \% Vol. & 0.05 & 0.05 & 0.05 \\
\hline \multirow[t]{2}{*}{ Salt Content \% Wt. } & 0.0012 & 0.0006 & 0.0006 \\
\hline & 3.97 & 4.54 & $4 . S$ \\
\hline Asphaltenes Content \% Wt. & 1.4 & & 1.85 \\
\hline Ash Content \% Wt. & 0.0070 & & \\
\hline Vanadium PPM & 23 & & 27.2 \\
\hline Nickel PPM & 6 & & 9 \\
\hline KUOP Characterization & 12.0 & & 12.0 \\
\hline \multicolumn{4}{|l|}{ Distillation } \\
\hline \multicolumn{4}{|l|}{ IBP c } \\
\hline Rec. @ 50.0 ${ }^{\circ} \mathrm{C}$ & 1.5 & 2.0 & 2 \\
\hline @ $75.0{ }^{\circ} \mathrm{C}$ & 4.5 & 4.0 & 4 \\
\hline @ $100.0^{\circ} \mathrm{C}$ & 9.0 & 9.5 & 8 \\
\hline @ $125.0^{\circ} \mathrm{C}$ & 14.0 & 14 & 14 \\
\hline @ $150.0^{\circ} \mathrm{C}$ & 22.5 & 19.5 & 19 \\
\hline @ $175.0^{\circ} \mathrm{C}$ & 29.5 & 25 & 24 \\
\hline @ $200.0^{\circ} \mathrm{C}$ & 36.0 & 30.5 & 29 \\
\hline @ $225.0^{\circ} \mathrm{C}$ & 41.5 & 36.5 & 35 \\
\hline @ $250.0^{\circ} \mathrm{C}$ & 46.5 & 39.5 & 39 \\
\hline @ $275.0^{\circ} \mathrm{C}$ & 50.0 & 43.5 & 43 \\
\hline @ $300.0^{\circ} \mathrm{C}$ & 52 & 48.0 & 47 \\
\hline Total Distil. \% Vol. & 57.0 & 50.0 & 49 \\
\hline
\end{tabular}




\section{Journal of Petroleum Researches \& Studies $\quad \begin{gathered}\mathrm{N}_{0} .2 \\ 2^{\text {nd. }} . \text { YEAR }\end{gathered}$}

Table-2

\begin{tabular}{|c|c|c|c|c|c|c|}
\hline \multicolumn{3}{|c|}{ Crude oil API. Gravity @ $15.6{ }^{\circ} \mathrm{C}=31.7$} & \multicolumn{2}{|c|}{ Crude oil Vis. $=7.94$} & \multicolumn{2}{|c|}{$25 / 11 / 08$} \\
\hline Sample & $\mathrm{LN}$ & $\mathrm{HN}$ & Ker & Gasoil & Diesel & $\mathrm{RC}$ \\
\hline API. Gravity @ $15.6{ }^{\circ} \mathrm{C}$ & 78.1 & 64.5 & 48.9 & 40.1 & 34.5 & 17.0 \\
\hline Flash point ${ }^{\circ} \mathrm{C}$ & & & 40.0 & 76.0 & 92.0 & 104.0 \\
\hline Color & +30 & +30 & +28 & 0.5 & & \\
\hline Doctor test & Ps & Ps & Ps & & & \\
\hline R.V.P.@ $37.8^{\circ} \mathrm{C}$ & & & & & & \\
\hline Pour point ${ }^{\circ} \mathrm{C}$ & & & & & & \\
\hline $\begin{array}{c}\text { Vis. @ } 100{ }^{\circ} \mathrm{C} \\
@ 50{ }^{\circ} \mathrm{C}\end{array}$ & & & & & 4.68 & $\begin{array}{l}22.82 \\
189.8\end{array}$ \\
\hline B.S. \& W & \multicolumn{6}{|c|}{$0.2 \%$ water and sediment } \\
\hline & & & & & & \\
\hline I.B.P & 43 & 55 & 138 & & & \\
\hline $10 \%$ & 52 & 74 & 160 & & & \\
\hline $20 \%$ & 60 & 80 & 168 & & & \\
\hline $30 \%$ & 74 & 86 & 174 & & & \\
\hline $40 \%$ & 78 & 92 & 180 & & & \\
\hline $50 \%$ & 82 & 100 & 188 & & & \\
\hline $60 \%$ & 90 & 106 & 196 & & & \\
\hline $70 \%$ & 98 & 112 & 202 & & & \\
\hline $80 \%$ & 108 & 120 & 212 & & & \\
\hline $90 \%$ & 141 & 136 & 222 & & & \\
\hline E.B.P & 152 & 172 & 255 & & & \\
\hline T.D. & 96 & 98 & 99 & & & \\
\hline RES. & 0.6 & 0.8 & 1.0 & & & \\
\hline
\end{tabular}




\section{Journal of Petroleum Researches \& Studies $\quad \begin{gathered}\mathrm{N}_{\mathrm{O}} .2 \\ 2^{\text {nd. }} \text { YEAR }\end{gathered}$}

Table-3

\begin{tabular}{|c|c|c|c|c|c|c|}
\hline \multicolumn{3}{|c|}{ Crude oil API. Gravity @ $15.6{ }^{\circ} \mathrm{C}=34.0$} & \multicolumn{2}{|c|}{ Crude oil Vis. $=5.51$} & \multicolumn{2}{|c|}{$26 / 11 / 08$} \\
\hline Sample & $\mathrm{LN}$ & $\mathrm{HN}$ & Ker & Gasoil & Diesel & $\mathrm{RC}$ \\
\hline API. Gravity @ $15.6{ }^{\circ} \mathrm{C}$ & 76.5 & 61.3 & 52.0 & 44.2 & 36.2 & 18.2 \\
\hline Flash point ${ }^{\circ} \mathrm{C}$ & & & 31.7 & 60.0 & 84.0 & 104.0 \\
\hline Color & +30 & +28 & +24 & 0.5 & & \\
\hline Doctor test & Ps & Ps & Ps & & & \\
\hline R.V.P.@ $37.8^{\circ} \mathrm{C}$ & & & & & & \\
\hline Pour point ${ }^{\circ} \mathrm{C}$ & & & & & & \\
\hline $\begin{array}{c}\text { Vis. @ } 100{ }^{\circ} \mathrm{C} \\
\text { @ } 50{ }^{\circ} \mathrm{C} \\
\end{array}$ & & & & & 3.77 & $\begin{array}{c}16.11 \\
117.07 \\
\end{array}$ \\
\hline B.S.\& W & \multicolumn{6}{|c|}{$0.2 \%$ water and sediment } \\
\hline & & & & & & \\
\hline I.B.P & 38 & 66 & 142 & & & \\
\hline $10 \%$ & 50 & 96 & 152 & & & \\
\hline $20 \%$ & 57 & 105 & 157 & & & \\
\hline $30 \%$ & 65 & 112 & 162 & & & \\
\hline $40 \%$ & 73 & 119 & 166 & & & \\
\hline $50 \%$ & 82 & 125 & 170 & & & \\
\hline $60 \%$ & 89 & 130 & 174 & & & \\
\hline $70 \%$ & 96 & 137 & 179 & & & \\
\hline $80 \%$ & 104 & 144 & 186 & & & \\
\hline $90 \%$ & 116 & 152 & 199 & & & \\
\hline E.B.P & 142 & 186 & 230 & & & \\
\hline T.D. & 96 & 98 & 99 & & & \\
\hline RES. & 0.6 & 0.8 & 1.0 & & & \\
\hline
\end{tabular}


Table-4

\begin{tabular}{|c|c|c|c|c|c|c|}
\hline \multicolumn{3}{|c|}{ Crude oil API. Gravity @ $15.6{ }^{\circ} \mathrm{C}=32.9$} & \multicolumn{2}{|c|}{ Crude oil Vis. $=7.18$} & \multicolumn{2}{|c|}{ 27/11/08 } \\
\hline Sample & LN & $\mathrm{HN}$ & Ker & Gasoil & Diesel & $\mathrm{RC}$ \\
\hline API. Gravity@15.6 ${ }^{\circ} \mathrm{C}$ & 77.1 & 61.2 & 49.3 & 41.3 & 33.6 & 17.6 \\
\hline Flash point ${ }^{\circ} \mathrm{C}$ & & & 37.8 & 68.0 & 94.0 & 95.0 \\
\hline Color & +30 & +28 & +26 & 0.5 & & \\
\hline Doctor test & Ps & Ps & Ps & & & \\
\hline \multicolumn{7}{|l|}{ R.V.P.@ $37.8^{\circ} \mathrm{C}$} \\
\hline \multicolumn{7}{|l|}{ Pour point ${ }^{\circ} \mathrm{C}$} \\
\hline $\begin{array}{c}\text { Vis. @ } 100{ }^{\circ} \mathrm{C} \\
\text { @ } 50{ }^{\circ} \mathrm{C}\end{array}$ & & & & & 5.9 & $\begin{array}{c}18.02 \\
133.86\end{array}$ \\
\hline B.S. \& W & \multicolumn{6}{|c|}{$0.2 \%$ water and sediment } \\
\hline & & & & & & \\
\hline I.B.P & 47 & 62 & 140 & & & \\
\hline $10 \%$ & 58 & 88 & 160 & & & \\
\hline $20 \%$ & 66 & 94 & 165 & & & \\
\hline $30 \%$ & 74 & 102 & 170 & & & \\
\hline $40 \%$ & 82 & 110 & 174 & & & \\
\hline $50 \%$ & 90 & 118 & 180 & & & \\
\hline $60 \%$ & 98 & 124 & 186 & & & \\
\hline $70 \%$ & 110 & 130 & 192 & & & \\
\hline $80 \%$ & 117 & 138 & 200 & & & \\
\hline $90 \%$ & 126 & 144 & 212 & & & \\
\hline E.B.P & 158 & 186 & 255 & & & \\
\hline T.D. & 96 & 98 & 99 & & & \\
\hline RES. & 0.6 & 0.8 & 1.0 & & & \\
\hline
\end{tabular}


Table-5

\begin{tabular}{|c|c|c|c|c|c|c|}
\hline \multicolumn{3}{|c|}{ Crude oil API. Gravity @ $15.6{ }^{\circ} \mathrm{C}=33.1$} & \multicolumn{2}{|c|}{ Crude oil Vis. $=6.88$} & \multicolumn{2}{|c|}{ 28/11/08 } \\
\hline Sample & LN & $\mathrm{HN}$ & Ker & Gasoil & Diesel & $\mathrm{RC}$ \\
\hline API. Gravity@15.6 ${ }^{\circ} \mathrm{C}$ & 75.3 & 60.4 & 49.3 & 42.0 & 34.7 & 17.1 \\
\hline Flash point ${ }^{\circ} \mathrm{C}$ & & & 42.2 & 70.0 & 85.0 & 104.0 \\
\hline Color & +30 & +27 & +26 & 0.5 & & \\
\hline Doctor test & Ps & Ps & Ps & & & \\
\hline R.V.P.@ $37.8^{\circ} \mathrm{C}$ & & & & & & \\
\hline Pour point ${ }^{\circ} \mathrm{C}$ & & & & & & \\
\hline $\begin{array}{c}\text { Vis. @ } 100{ }^{\circ} \mathrm{C} \\
@ 50{ }^{\circ} \mathrm{C}\end{array}$ & & & & & 5.74 & $\begin{array}{l}18.90 \\
133.98\end{array}$ \\
\hline B.S.\&W & \multicolumn{6}{|c|}{$0.2 \%$ water and sediment } \\
\hline & & & & & & \\
\hline I.B.P & 45 & 66 & 147 & & & \\
\hline $10 \%$ & 54 & 94 & 169 & & & \\
\hline $20 \%$ & 62 & 102 & 174 & & & \\
\hline $30 \%$ & 69 & 108 & 180 & & & \\
\hline $40 \%$ & 76 & 114 & 186 & & & \\
\hline $50 \%$ & 83 & 120 & 192 & & & \\
\hline $60 \%$ & 90 & 125 & 198 & & & \\
\hline $70 \%$ & 100 & 131 & 207 & & & \\
\hline $80 \%$ & 109 & 138 & 212 & & & \\
\hline $90 \%$ & 121 & 148 & 222 & & & \\
\hline E.B.P & 150 & 183 & 249 & & & \\
\hline T.D. & 96 & 98 & 99 & & & \\
\hline RES. & 0.6 & 0.8 & 1.0 & & & \\
\hline
\end{tabular}

As we said before in the abstract our experiment consists of two stages:-

\section{Stage before changing operating conditions:-}

In this stage the following operating conditions system has been used in Daura crude distilla- tion units before the improvement time, as listed in tables (6, 7). 
Table-6

\begin{tabular}{|c|c|}
\hline \multicolumn{2}{|c|}{ Production quantities } \\
\hline Cdu. No.2 & Before (BBL/hr) \\
\hline Crude oil feed(API=32.7) & 800 \\
\hline HN. & $60-70$ \\
\hline Ker. & $70-90$ \\
\hline L.G.O. & $(60-40)+$ gland oil \\
\hline H.G.O. & 25 \\
\hline RC. & - \\
\hline
\end{tabular}

Table-7

\begin{tabular}{|c|c|}
\hline \multicolumn{2}{|c|}{ Operating conitions } \\
\hline Cdu. No.2 & Before \\
\hline Top temp. $\left({ }^{\circ} \mathrm{F}\right)$ & $200-215$ \\
\hline Bottom temp. $\left({ }^{\circ} \mathrm{F}\right)$ & $630-640$ \\
\hline Upper Reflux draw-off temp. $\left({ }^{\circ} \mathrm{F}\right)$ & $250-260$ \\
\hline $\begin{array}{l}\text { Upper Reflux draw-off quantity. } \\
\text { (BBL/hr) }\end{array}$ & Equal to feed \\
\hline Lower Reflux (in) temp. $\left({ }^{\circ} \mathrm{F}\right)$ & $160-180$ \\
\hline $\begin{array}{l}\text { Lower Reflux draw-off quantity. } \\
\text { (BBL/hr) }\end{array}$ & $33 \%$ of feed \\
\hline
\end{tabular}

Also chromatography analysis for Heavy Naphtha (gasoline) has been done in Daura laboratory before the time of experiment, as shown in the figures $(1,2)$.

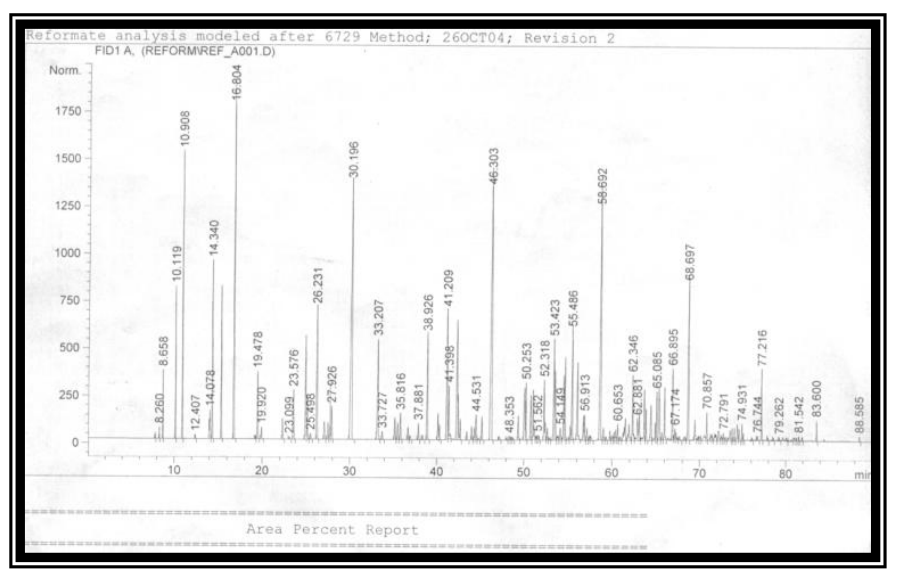

Fig.1

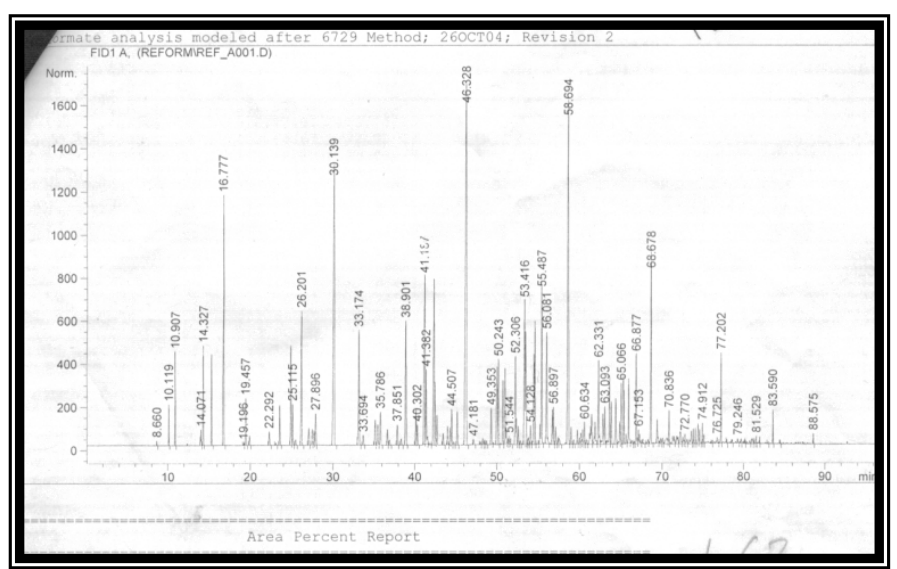

Fig.2 


\section{Stage after changing operating} conditions (Experiment

\section{Time):-}

In this stage the new operating conditions system has been adopted as we got it from simulation program (HYSYS) and several analyses for all products have been done.

\section{$\underline{\text { Results }}$}

New operating conditions and products analysis tabulated below in tables $(8,9,10,11,12)$ and distillation curves in figures $(4,5,6,7,8,9,10)$.

Table- 8

\begin{tabular}{|c|c|}
\hline \multicolumn{2}{|c|}{ New product quantities } \\
\hline Cdu. & After (BBL/hr) \\
\hline Crude oil feed (API=32.7) & 800 \\
\hline HN. & $120-130$ \\
\hline Ker. & $90-115$ \\
\hline L.G.O. & $105+$ gland oil \\
\hline H.G.O. & Increased \\
\hline RC. & Decreased \\
\hline
\end{tabular}

Table-9

\begin{tabular}{|l|c|}
\hline \multicolumn{2}{|c|}{ New operating condition system } \\
\hline \multicolumn{1}{|c|}{ Cdu. } & After \\
\hline Top temp. $\left({ }^{\circ} \mathrm{F}\right)$ & $226-228$ \\
\hline Bottom temp. $\left({ }^{\circ} \mathrm{F}\right)$ & $600-610$ \\
\hline Upper Reflux draw-off temp. $\left({ }^{\circ} \mathrm{F}\right)$ & $280-290$ \\
\hline $\begin{array}{l}\text { Upper Reflux draw-off quantity. } \\
\text { (BBL/hr) }\end{array}$ & $85-90 \%$ of feed \\
\hline Lower Reflux (in) temp. $\left({ }^{\circ} \mathrm{F}\right)$ & $240-250$ \\
\hline $\begin{array}{l}\text { Lower Reflux draw-off quantity. } \\
(\mathrm{BBL} / \mathrm{hr})\end{array}$ & $24 \%$ of feed \\
\hline
\end{tabular}


Table-10

\begin{tabular}{|c|c|c|c|c|c|c|}
\hline \multicolumn{3}{|c|}{ Crude oil API. Gravity @ $15.6{ }^{\circ} \mathrm{C}=31.7$} & \multicolumn{2}{|c|}{ Crude oil Vis. $=7.92$} & \multicolumn{2}{|c|}{$02 / 12 / 08$} \\
\hline Sample & $\mathbf{L N}$ & HN & Ker & Gasoil & Diesel & $\mathbf{R C}$ \\
\hline API. Gravity @ $15.6^{\circ} \mathrm{C}$ & 77.2 & 61.6 & 48.6 & 41.3 & 36.0 & $\mathbf{1 7 . 0}$ \\
\hline Flash point ${ }^{\circ} \mathrm{C}$ & & & 43.3 & 74.0 & 86.0 & 104.0 \\
\hline Color & $+\mathbf{3 0}$ & +28 & +27 & 0.5 & & \\
\hline Doctor test & Ps & Ps & Ps & & & \\
\hline R.V.P. @ $37.8^{\circ} \mathrm{C}$ & & & & & & \\
\hline Pour point ${ }^{\circ} \mathrm{C}$ & & & & & & \\
\hline $\begin{array}{c}\text { Vis. @ } 100{ }^{\circ} \mathrm{C} \\
@ 50^{\circ} \mathrm{C}\end{array}$ & & & & & 3.02 & \begin{tabular}{|c|}
20.19 \\
170.14 \\
\end{tabular} \\
\hline B.S. \& W & \multicolumn{6}{|c|}{$0.2 \%$ water and sediment } \\
\hline I.B.P & 42 & 64 & 150 & & & \\
\hline $10 \%$ & 51 & 100 & 165 & & & \\
\hline $20 \%$ & 60 & 115 & 170 & & & \\
\hline $30 \%$ & 68 & 125 & 175 & & & \\
\hline $40 \%$ & 76 & 130 & 180 & & & \\
\hline $50 \%$ & 86 & 135 & 186 & & & \\
\hline $60 \%$ & 94 & 140 & 190 & & & \\
\hline $70 \%$ & 104 & 145 & 200 & & & \\
\hline $80 \%$ & 112 & 150 & 210 & & & \\
\hline $90 \%$ & 121 & 160 & 220 & & & \\
\hline E.B.P & 150 & 180 & 248 & & & \\
\hline T.D. & 96 & 98 & 99 & & & \\
\hline RES. & 0.6 & 0.8 & 1.0 & & & \\
\hline
\end{tabular}


Table-11

\begin{tabular}{|c|c|c|c|c|c|c|}
\hline \multicolumn{3}{|c|}{ Crude oil API. Gravity @ $15.6{ }^{\circ} \mathrm{C}=32.0$} & \multicolumn{2}{|c|}{ Crude oil Vis. $=7.73$} & \multicolumn{2}{|c|}{$03 / 12 / 08$} \\
\hline Sample & LN & $\mathrm{HN}$ & Ker & Gasoil & Diesel & $\mathrm{RC}$ \\
\hline API. Gravity@15.6 ${ }^{\circ} \mathrm{C}$ & 77.0 & 62.7 & 48.6 & 39.5 & 33.2 & 16.4 \\
\hline Flash point ${ }^{\circ} \mathrm{C}$ & & & 41.1 & 80.0 & 90.0 & 104.0 \\
\hline Color & +30 & +28 & +26 & 0.5 & & \\
\hline Doctor test & Ps & Ps & Ps & & & \\
\hline R.V.P.@ $37.8^{\circ} \mathrm{C}$ & & & & & & \\
\hline Pour point ${ }^{\circ} \mathrm{C}$ & & & & & & \\
\hline $\begin{array}{c}\text { Vis. @ } 100{ }^{\circ} \mathrm{C} \\
@ 50{ }^{\circ} \mathrm{C}\end{array}$ & & & & & 5.48 & $\begin{array}{c}25.53 \\
241.01 \\
\end{array}$ \\
\hline B.S.\& W & \multicolumn{6}{|c|}{$0.2 \%$ water and sediment } \\
\hline & & & & & & \\
\hline I.B.P & 42 & 60 & 138 & & & \\
\hline $10 \%$ & 50 & 88 & 160 & & & \\
\hline $20 \%$ & 56 & 100 & 166 & & & \\
\hline $30 \%$ & 62 & 108 & 172 & & & \\
\hline $40 \%$ & 70 & 115 & 180 & & & \\
\hline $50 \%$ & 78 & 122 & 186 & & & \\
\hline $60 \%$ & 86 & 130 & 192 & & & \\
\hline $70 \%$ & 92 & 138 & 200 & & & \\
\hline $80 \%$ & 102 & 142 & 212 & & & \\
\hline $90 \%$ & 118 & 150 & 224 & & & \\
\hline E.B.P & 148 & 178 & 252 & & & \\
\hline T.D. & 96 & 98 & 99 & & & \\
\hline RES. & 0.6 & 0.8 & 1.0 & & & \\
\hline
\end{tabular}


Table-12

\begin{tabular}{|c|c|c|c|c|c|c|}
\hline \multicolumn{3}{|c|}{ Crude oil API. Gravity @ $15.6{ }^{\circ} \mathrm{C}=33.3$} & \multicolumn{2}{|c|}{ Crude oil Vis. $=6.78$} & \multicolumn{2}{|c|}{$04 / 12 / 08$} \\
\hline Sample & $\mathrm{LN}$ & $\mathrm{HN}$ & Ker & Gasoil & Diesel & $\mathrm{RC}$ \\
\hline API. Gravity@15.6 ${ }^{\circ} \mathrm{C}$ & 77.6 & 62.4 & 50.3 & 41.5 & 34.3 & 17.1 \\
\hline Flash point ${ }^{\circ} \mathrm{C}$ & & & 35.6 & 86.0 & 100.0 & 92.0 \\
\hline Color & +30 & +28 & +26 & 0.5 & & \\
\hline Doctor test & Ps & Ps & Ps & & & \\
\hline R.V.P. @ $37.8^{\circ} \mathrm{C}$ & & & & & & \\
\hline Pour point ${ }^{\circ} \mathrm{C}$ & & & & & & \\
\hline $\begin{array}{c}\text { Vis. @ } 100{ }^{\circ} \mathrm{C} \\
\text { @ } 50{ }^{\circ} \mathrm{C}\end{array}$ & & & & & 5.86 & $\begin{array}{c}26.58 \\
242.36\end{array}$ \\
\hline B.S. \& W & \multicolumn{6}{|c|}{$0.2 \%$ water and sediment } \\
\hline & & & & & & \\
\hline I.B.P & 36 & 55 & 140 & & & \\
\hline $10 \%$ & 48 & 82 & 162 & & & \\
\hline $20 \%$ & 56 & 90 & 168 & & & \\
\hline $30 \%$ & 66 & 96 & 172 & & & \\
\hline $40 \%$ & 75 & 106 & 176 & & & \\
\hline $50 \%$ & 84 & 114 & 180 & & & \\
\hline $60 \%$ & 92 & 121 & 185 & & & \\
\hline $70 \%$ & 100 & 129 & 190 & & & \\
\hline $80 \%$ & 110 & 138 & 196 & & & \\
\hline $90 \%$ & 123 & 149 & 206 & & & \\
\hline E.B.P & 156 & 184 & 238 & & & \\
\hline T.D. & 96 & 98 & 99 & & & \\
\hline RES. & 0.6 & 0.8 & 1.0 & & & \\
\hline
\end{tabular}




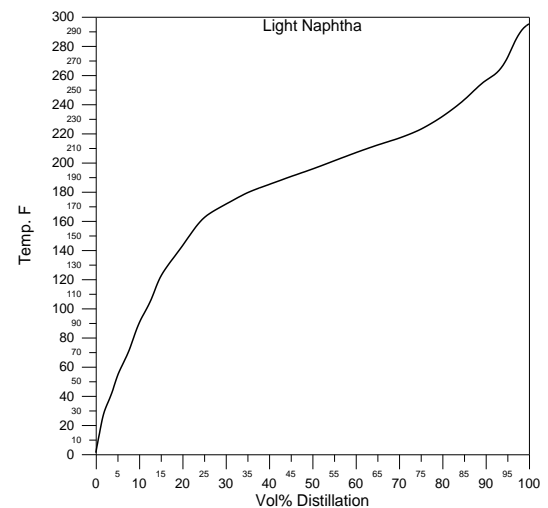

Fig-3

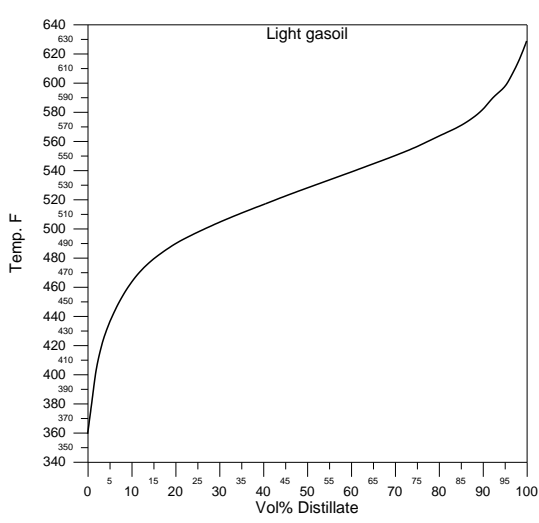

Fig-6

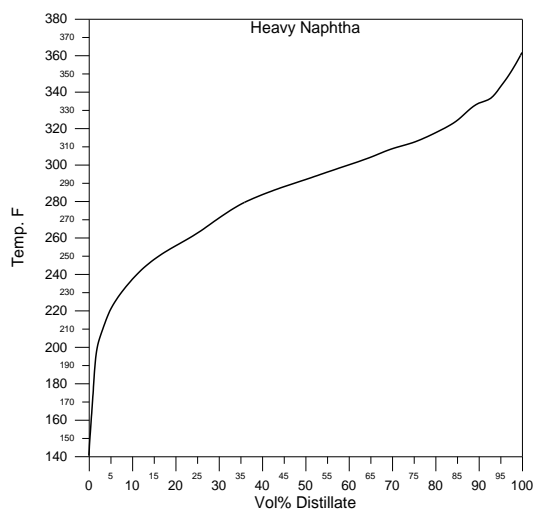

Fig-4

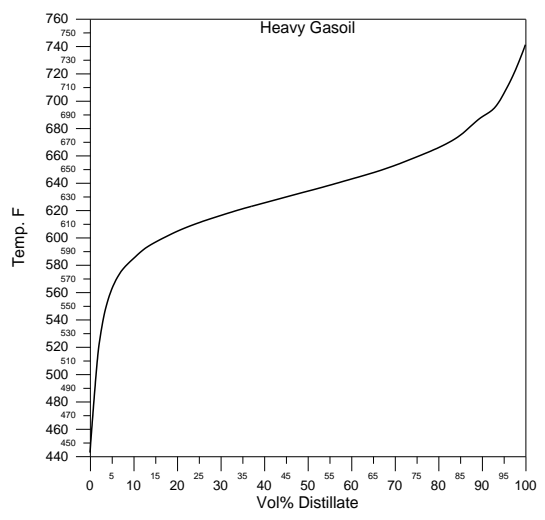

Fig-7

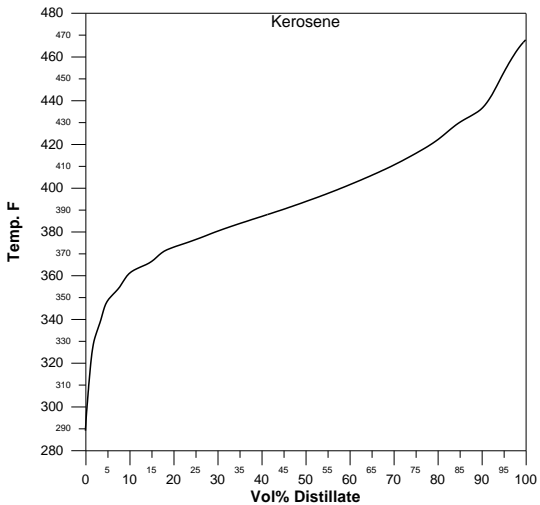

Fig-5

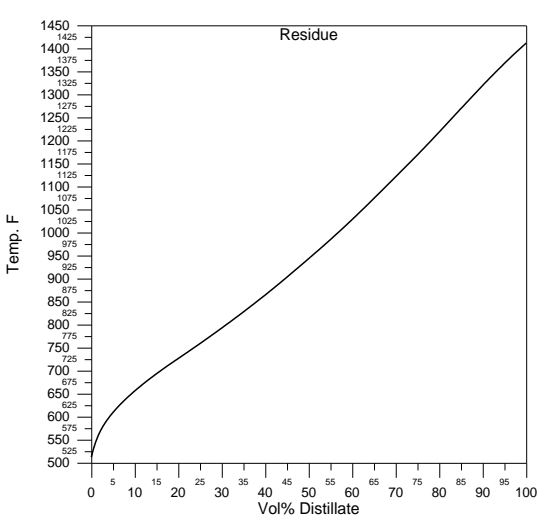

Fig-8

Table-13

\begin{tabular}{|c|c|c|c|c|}
\hline \multicolumn{4}{|c|}{$\mathbf{M}^{3} /$ year } & \multicolumn{2}{|c|}{$\mathbf{M}^{3} /$ year } & Product \\
\hline$\%$ & $\begin{array}{c}\text { Annual } \\
\text { increasing }\end{array}$ & $\begin{array}{c}\text { Amount of } \\
\text { product after } \\
\text { experiment }\end{array}$ & $\begin{array}{c}\text { Amount of } \\
\text { product before } \\
\text { experiment }\end{array}$ & \\
\hline $100 \%$ & 82416.534 & 164833.06 & 82416.534 & HN \\
\hline
\end{tabular}




\section{$\underline{\text { Discussion }}$}

As a result of experiment the following points have been noticed:-

1. Water accumulation in water separator drum decreased to $(90 \%)$ and that means no water will be present in the top trays and subsequently corrosion action due to $(\mathrm{HCl})$ will be decreased.

2. End and initial boiling point of heavy naphtha, products and PONA distribution becomes stable despite withdrawing quantity increasing.

3. Decreasing the quantity of reduced crude (RC) despite reducing of furnace outlet temperature from $\left(640{ }^{\circ} \mathrm{F}\right)$ to $\left(600-620{ }^{\circ} \mathrm{F}\right)$ and decreasing stripping steam, which means annual fuel saving.

4. Total amount of products after this improvement increased more than (100 $\mathrm{bbl} / \mathrm{hr}$ ) for crude oil with API equal to (32.7).

5. Presence of condensed water in the upper section of the crude distillation tower particularly in the upper reflux trays plays as a water trap and decrease fractionation efficiency, in addition this makes the tower under the risk of high pressure due to water vaporization in case of lack or becoming half or less original quantity of upper reflux.

Now becomes possible to supply reforming unit with heavy naphtha continuously depending on only one unit but it was impossible before the experiment due to low production rate, see table (13). 


\section{Journal of Petroleum Researches \& Studies $\quad \begin{gathered}\mathrm{N}_{\mathrm{O}} .2 \\ 2^{\text {nd. }} \mathrm{YEAR}\end{gathered}$}

\section{Conclusion}

1. Presence of water condensate in the tower will decrease the fractionation efficiency.

2. Corrosion becomes less in the top section of the distillation tower.
3. Using simulation programs technology can improve the operation process.

4. It is possible to improve operation conditions system for crude distillation units particularly if they are old.

\section{References}

1. Simulation program (HYSYS).

2. Donald M. Little/ Catalytic Reforming/ page-25. 
الاسم : عائـــــــابر عمران طعمة

$$
\text { موقع العمل :شركة مصافي الوسط / بغداد الوظيفي : مهند كيمياوي اقدم }
$$

التحصيل العلمي : ماجستير في تطوير عمل وحدات التكرير الحيوي /روسيا /جامعة اوفا 